\title{
Hausdorff dimension of random attractor for stochastic Navier-Stokes-Voight equations and primitive equations
}

\author{
Chengfeng Sun and Hongjun Gao \\ Communicated by Yuncheng You, received October 7, 2009.
}

\begin{abstract}
We study the three dimensional stochastic Navier-Stokes-Voight model of viscoelastic incompressible fluid and three dimensional stochastic Primitive Equations with additive noise. In [12] and [11], we showed that the long time dynamics is captured by a random attractor, along this line, in this paper, we show that the Hausdorff dimension of attractor is an invariant random variable, and it is bounded by $d$, provided the random dynamics system contracts $d$-dimensional volumes exponentially fast.
\end{abstract}

\section{Contents}

1. Introduction 307

2. Technical Preliminaries 308

3. Finite Hausdorff Dimension for SNSV 309

4. Finite Dimension of the Attractor for SPEs 317

References $\quad 325$

\section{Introduction}

The need to take stochastic effects into account for modeling complex systems has now become widely recognized. Stochastic partial differential equations arise naturally as mathematical models for nonlinear macroscopic dynamics under random influences. It is thus desirable to understand the impact of such random influences on the system evolution $[\mathbf{7}],[\mathbf{2 1}]$ and $[\mathbf{2 3}]$. The theory of random attractors is becoming very useful for the study of the asymptotic behavior of dissipative $86 \mathrm{~A} 10$.

2000 Mathematics Subject Classification. Primary 35Q99, 60H15; Secondary 76M35, 86A05,

Key words and phrases. Hausdorff dimension, Navier-Stokes-Voight equations, primitive equations.

Partially supported by a China NSF Grant No. 10871097 and National Basic Research Program of China (973 Program) No. 2007CB814800. 
random dynamical systems, Crauel and Flandoli [5] and Crauel, Debussche and Flandoli [4] developed a theory for the existence of random attractors for stochastic systems that closely parallels the deterministic theory. A random attractor is a random invariant compact set which attracts every trajectory as time goes to infinite. Moreover, it can be proved that, in some cases, the random attractor has finite Hausdorff dimension. It seems that the asymptotic behavior of the random dynamical systems is governed by a finite number of degrees of freedom. Crauel and Flandoli [6] developed a method for bounding the Hausdorff dimension of attractors for certain systems, and their techniques required the noise to be bounded; Debussche [8] used a random squeezing property (cf. [10]) to bound the Hausdorff dimension without the assumption of bounded noise, a technique generalized to treat the fractal dimension by Langa [19]. However, the best bounds in the deterministic theory come not from a use of the squeezing property, but from the method involving Lyapunov exponents developed by Constantin, Foias and Temam $[\mathbf{3}]$. It is this method that was adapted to the stochastic case by Debussche [9] to obtain an upper bound on the Hausdorff dimension. In his paper he remarks that the same arguments could be used to obtain a bound on the fractal dimension of such sets.

In this paper, we study the three dimensional Stochastic Navier-Stokes-Voight model of viscoelastic incompressible fluid (SNSV) and three dimensional Stochastic Primitive Equations (SPEs) with additive noise. In [12] and [11], we showed that the long time dynamics is captured by a random attractor. Using the method in [6], we show that the Hausdorff dimension is an invariant random variable, and it is bounded by $d$, provided the random dynamics system contracts $d$-dimensional volumes exponentially fast.

This paper is organized as follows. Some technical preliminaries to obtain the finite Hausdorff dimension is in $\S 2$. Finite Hausdorff dimension of random attractor for SNSV is showed in $§ 3$. Finally, finite Hausdorff dimension of random attractor for SPEs is showed in $\S 4$.

\section{Technical Preliminaries}

In order to obtain the finite Hausdorff dimension, we should need some technical preliminaries.

DEFINITION 2.1. The Hausdorff dimension $\operatorname{dim}_{H}(Y)$ of a subset $Y$ of a metric space is

$$
\operatorname{dim}_{H}(Y)=\inf \left\{s \geq 0: \mu_{H}(Y, s)=0\right\}=\sup \left\{s \geq 0: \mu_{H}(Y, s)>0\right\},
$$

where $\mu_{H}(Y, s)$ is the s-dimensional Hausdorff measure of $Y$ (see Definition in Temam $[\mathbf{2 2}])$.

We next show that volume contraction of an random dynamical system (RDS) on a random compact invariant set implies that the Hausdorff of this set is finite.

Suppose $S$ is a continuous RDS on a separable Hilbert space $H$ with norm $|\cdot|$. $L$ is a bounded linear operator on $H . \wedge^{d} L$ denotes the $d$-fold exterior product of $L$, and $\|\cdot\|$ denotes the operator norm defined by

$$
\left\|\wedge^{d} L\right\|_{\mathscr{L}\left(H^{d}, \wedge^{d} H\right)}=\sup _{\varphi_{1}, \ldots, \varphi_{d} \in H ;\left|\varphi_{i}\right|_{H} \leq 1, \forall i}\left|\left(\wedge^{d} L\right)\left(\varphi_{1}, \ldots, \varphi_{d}\right)\right|_{\wedge^{d} H}
$$


where

$$
\left(\wedge^{d} L\right)\left(\varphi_{1}, \ldots, \varphi_{d}\right)=L \varphi_{1} \wedge \cdots \wedge L \varphi_{d}
$$

Here we briefly recall the definitions of the exterior product of spaces and vectors in the case of Hilbert spaces, for further details the reader is referred to R. Temam $[22]$.

Suppose $w \mapsto X(w)$ is a compact strictly invariant set for $S$. We assume that $S$ is weakly differentiable on $X$ in the sence that (for $\mathbb{P}$-almost all $w$ ) for every $u \in X(w)$ and $t>0$ there exists a linear map $D_{u} S(t, w): H \rightarrow H$ such that

$$
\left.\begin{array}{r}
g_{\delta}(t, w)=\sup \left\{\frac{\left|S(t, w) u-S(t, w) v-D_{u} S(t, w)(u-v)\right|}{|u-v|}:\right. \\
u, v \in X(w), \quad|u-v| \leq \delta
\end{array}\right\}
$$

is finite for any $\delta>0$, and converges to zero $\mathbb{P}$-a.s. as $\delta \rightarrow 0$ for any $t>0$ fixed. In case $S$ is Frécher differentiable in $x$, the map $D_{x} S(t, w)$ is the differential. Put

$$
\gamma_{1}(t, w)=\sup _{u \in X(w)}\left\|D_{u} S(t, w)\right\|, \quad \Gamma_{d}(t, w)=\sup _{u \in X(w)}\left\|\wedge^{d} D_{u} S(t, w)\right\| .
$$

The following is the main theorem to obtain the finite Hausdorff dimension.

ThEOREM 2.2. [6] Suppose $S$ is an RDS on a separable Hilbert space H. Let $w \mapsto X(w)$ be a random compact set, strictly invariant for $S$, and suppose $S$ is weakly differentiable on $X$ in the sense of (2.1). Suppose that there exist $d$ and $t_{0}>0$ such that $\mathbb{P}$-a.s.

$$
\sup _{u \in X(w)}\left\|\wedge^{d} D_{u} S\left(t_{0}, w\right)\right\|=\Gamma_{d}\left(t_{0}, w\right)<1
$$

Suppose further that

- $\gamma_{1}(t, w) \in L^{\infty}(\Omega ; \mathbb{P})$ for every $t \geq 0$.

- $g_{\delta}(t, w) \in L^{\infty}(\Omega ; \mathbb{P})$ for every $t \geq 0$.

- $g_{\delta}(t, w)$ converges to zero in $\in L^{\infty}(\Omega ; \mathbb{P})$ for every $t>0$.

Then the Hausdorff dimension of $X$ satisfies $\operatorname{dim}_{H}(X) \leq d$.

\section{Finite Hausdorff Dimension for SNSV}

3.1. Existence of the Attractor. We consider the three-dimensional Stochastic Navier-Stokes-Voight (SNSV) system of equations

$$
\begin{aligned}
v_{t}-\nu \Delta v-\alpha^{2} \Delta v_{t}+(v \cdot \nabla) v+\nabla p & =f(x)+n(t), \quad x \in D, \quad t \in \mathbb{R} \\
\operatorname{div} v=0, x \in D ; v(x, t) & =0, \quad x \in \partial D \\
v(x, 0) & =v_{0}(x), \quad x \in D
\end{aligned}
$$

where $D \subset \mathbb{R}^{3}$ is a bounded domain with sufficiently smooth boundary $\partial D, v=$ $v(x, t)$ is the velocity vector field, $p$ is the pressure, $\nu>0$ is the kinematic viscosity, $\alpha$ is a length scale parameter characterizing the elasticity of the fluid, $f$ is a given force field, $n(t)$ are noise forcing terms and are taken as $\frac{\partial}{\partial t} W(t)$, here we assume that $W(t)$ is an $H$-value infinite dimensional Brownian motion of the form

$$
W(t)=\sum_{j=1}^{\infty} \sigma_{j} \beta_{j}(t) e_{j},
$$


where $\beta_{1}, \beta_{2}, \ldots$ is a sequence of independent standard Brownian motions on a complete probability space $(\Omega, \mathcal{F}, P)$ (with expectation denoted by $\mathbb{E}$ ) and $\left(e_{j}\right)_{j \in \mathbb{N}}$ is an orthonormal basis of a function space $H$ which we'll give below.

The system (1.1)-(1.3) models the stochastic dynamical of a Kelvin-Voight viscoelastic incompressible fluid. The determining model (for $n(t)=0$ ) was introduced in [20] as a model of motion of linear viscoelastic fluids. The authors of $[\mathbf{1}]$ proposed the inviscid simplified Bardina model (or equivalently the inviscid NSV equations) as a non-viscous (inviscid) regularization of the 3D Euler equations, subject to periodic boundary conditions. Motivated by this observation the system (1.1)-(1.3) was also proposed in [1] as a regularization, for small values of $\alpha$, of the 3D NavierStokes (NS) equations for the purpose of direct numerical simulations for both the periodic and the no-slip Dirichlet boundary conditions. In [20] it is shown that the initial boundary value problem (1.1)-(1.3) has a unique weak solution. In [15] and [16], it is shown that the semigroup generated by the problem (1.1)-(1.3) has a finite dimensional global attractor. Recently Cao, Lunasin, Titi [1] have obtained the global regularity of the inviscid version of 3D Kelvin-Voight model. The Gevrey regularity of the global attractor and determining modes for the 3D Navier-StokesVoight equations (Kelvin-Voight equation) are given by [17]-[18].

In this section, as in [12], we use the following notations in the mathematical theory for SNSV equations:

- $\quad L^{p}(D), 1 \leq p \leq \infty$, and $H^{s}(D)$ are the usual Lebesgue and Sobolev spaces, respectively.

- For $v=\left(v_{1}, v_{2}, v_{3}\right)$ and $u=\left(u_{1}, u_{2}, u_{3}\right)$, we denote by

$$
(u, v)=\sum_{j=1}^{3}\left(u_{j}, v_{j}\right)_{L_{2}(D)}, \quad\|v\|^{2}=\sum_{j=1}^{3}\left\|v_{j}\right\|_{L_{2}(D)}^{2}, \quad\|\nabla v\|^{2}=\sum_{i, j=1}^{3}\left\|\partial_{i} v_{j}\right\|_{L_{2}(D)}^{2} .
$$

- We set

$$
\mathcal{V}:=\left\{v \in\left(C_{0}^{\infty}(D)\right)^{3} ; \nabla \cdot v=0\right\} .
$$

- $\quad H$ is the closure of the set $\mathcal{V}$ in $\left(L_{2}(D)\right)^{3}$ topology.

- $\quad P$ is the Helmholz-Leray orthogonal projection in $\left(L_{2}(D)\right)^{3}$ onto the space $H$.

- $A:=-P \Delta$ is the Stokes operator subject to the no-slip homogeneous Dirichlet boundary condition with the domain $\left(H_{2}(D)\right)^{3} \cap V$. The operator $A$ is a selfadjoint positively definite operator in $H$, whose inverse $A^{-1}$ is a compact operator from $H$ into $H$. Thus it has an orthonormal system of eigenfunctions $\left\{e_{j}\right\}_{j=1}^{\infty}$ of A.

- We denote by $\left\{\lambda_{j}\right\}_{j=1}^{\infty}, 0<\lambda_{1} \leq \lambda_{2} \leq \cdots$, the eigenvalues of the Stokes operator $A$ corresponding to eigenfunctions $\left\{e_{j}\right\}_{j=1}^{\infty}$, repeated according to their multiplicities.

- $\quad V_{s}:=D\left(A^{s / 2}\right),\|v\|_{s}=\left\|A^{\frac{s}{2}}\right\|, s \in \mathbb{R} . V=V_{1}=\left(H_{0}^{1}(D)\right)^{3} \cap H$ is the Hilbert space with the norm, thanks to the Poincaré inequality. Clearly $V_{0}=H$.

- For $u, v, w \in \mathcal{V}$ we define the following bilinear form

$$
B(u, v):=P((u \cdot \nabla) v) \text { and the trilinear form } b(u, v, w)=(B(u, v), w) .
$$

The bilinear form $B(\cdot, \cdot)$ can be extended as a continuous operator $B: V \times V \rightarrow$ $V^{\prime}$, where $V^{\prime}$ is the dual of $V$ (see, e.g., $[\mathbf{2}]$ ).

- $\quad$ For each $u, v, w \in V$,

$$
b(u, v, v)=0 \text { and } b(u, v, w)=-b(u, w, v) .
$$


In [12], for convenience, we noted $\alpha=1$ and considered the auxiliary equation

$$
(I+A) h_{t}+(\nu+\tau)(I+A) h=\sum_{j=1}^{\infty} \sigma_{j} \frac{d \beta_{j}(t)}{d t} e_{j}
$$

since $(I+A)$ is positive self-adjoint operators, $(I+A)^{-1}$ exists and is compact, and $(I+A)^{-1} e_{j}=\left(1+\lambda_{j}\right)^{-1} e_{j}$. Equation (3.5) equivalently the following OrnsteinUhlenbeck equation

$$
h_{t}+(\nu+\tau) h=\frac{d n_{1}(t)}{d t}
$$

where $n_{1}(t)=\sum_{j=1}^{\infty}\left(1+\lambda_{j}\right)^{-1} \sigma_{j} \beta_{j}(t) e_{j}$. Since $W(t)$ is an $H$-valued Wiener process, we obtained that the process

$$
h(t)=\int_{-\infty}^{t} e^{-(t-s)(\nu+\tau)} d n_{1}(s)
$$

is a stationary ergodic solution with continuous trajectories, take values in $D(A)$. In particular,

$$
\mathbb{E}\|A h(t)\|^{2}=\sum_{j=1}^{\infty} \frac{\lambda_{j}^{2} \sigma_{j}^{2}}{2(\nu+\tau)\left(1+\lambda_{j}\right)^{2}}<\infty .
$$

In a similar way, we have $\mathbb{E}\|h\|^{2}, \mathbb{E}\left\|A^{1 / 2} h\right\|^{2}<\infty$. However, when $\alpha \neq 1$, above results are also correct.

Then we can transform the SNSV to a random partial differential equation. The main advantage of this method is that we can now solve our equation $\omega$-wise, because of the absence of stochastic integrals. In this way we avoid the issue of exceptional sets of the solution depending on the initial condition.

Using the change of variable $u(t)=v(t)-h(t)$, then $u(t, x)$ satisfies the equation (which depends on a random parameter):

$$
\begin{aligned}
u_{t}+\nu A u+A u_{t}+B(u+h, u+h) & =f(x)+(\nu+\tau) h+\tau A h \\
u(0, x) & =v_{0}-h_{0} .
\end{aligned}
$$

Now we give the well-posedness of the equation (3.8)-(3.9).

Theorem 3.1. (see $[\mathbf{1 2}]$ ) Assume that $t_{0} \in \mathbb{R}$ and $u_{0} \in V$, for $\mathbb{P}$-almost every $\omega \in \Omega$ the following holds:

(i) there exist a unique solution of (3.8)-(3.9) on the time interval $\left[t_{0}, \infty\right)$ and satisfies $u \in C\left(\left[t_{0}, \infty\right), V\right)$.

(ii) denoting such solution by $u\left(t, \omega ; t_{0}, u_{0}\right)$, the mapping $u_{0} \mapsto u\left(t, \omega ; t_{0}, u_{0}\right)$ is continuous for all $t \geq t_{0}$.

Then having the mapping $v_{0} \mapsto u\left(t, w ; 0, u_{0}\right)+h(t, w)$, one can define a stochastic flow $S(t, w)$ by

$$
S(t, w) v_{0}=u\left(t, w ; 0, u_{0}\right)+h(t, w) .
$$

Also in [12], using uniform estimates and asymptotic compactness, we showed that the long time dynamics is captured by a random attractor. 
THEOREM 3.2. (see [12]) Let $K(w)$ be the ball in $V$ of radius $R_{0}(w)=r_{0}(w)^{\frac{1}{2}}+$ $\|h(0, w)\|_{1}$, where

$$
\begin{aligned}
r_{0}(w)= & C \int_{-\infty}^{0}\left(g(\sigma)+C\|f\|^{2}\right) \exp \left(-\int_{\sigma}^{0}\left[d_{0}-C\left(\|h(\varsigma)\|+\|h(\varsigma)\|_{1}\right)\right] d \varsigma\right) d \sigma \\
& +C \sup _{s \in(-\infty, 0]}\left(\left[\|h(s)\|^{2}+\|h(s)\|_{1}^{2}\right] \exp \left(s \frac{d_{0}}{2}\right)\right)+1, \\
d_{0}= & \frac{\nu}{2} \min \left\{1, \lambda_{1}\right\}, \\
g(t)= & C\left(\|h\|^{2}+\|h\|_{1}^{2}+\|h\|_{1}^{3}\|h\|\right),
\end{aligned}
$$

such that for any $B$ bounded in $V$, there exists $s_{1}(w, B)$, for $s<s_{1}(w, B)$, we have

$$
S(0, s ; w) B \subset K(w), \quad \mathbb{P}-\text { a.s. }
$$

and the stochastic flow $S$ is asymptotically compact in $V$ for $\mathbb{P}$-a.s., thus we obtain a random attractor $\mathcal{A}(\omega)$ which attracts every bounded nonrandom set $B \subset V$.

3.2. Volume Contraction. Consider the differential equation of (3.1) on $V$

$$
\begin{aligned}
& U_{t}(t, w)+\alpha^{2} A U_{t}(t, w)=F^{\prime}(v(t)) U(t), \\
& U(0, w)=\xi \in V,
\end{aligned}
$$

where

$$
F^{\prime}(v(t)) U=-\nu A U(t, w)-B\left(S(t, w) v_{0}, U(t, w)\right)-B\left(U(t, w), S(t, w) v_{0}\right) .
$$

The existence of solution $U \in C([0, T] ; V)$ for all $T>0$ can be established, path by path, as in the deterministic case in [18]. One can show that the flow $S(t, w)$ associated with (3.12)-(3.13) has the property that $v \mapsto S(t, w) v$ is differentiable and for each $v_{0}$ and $\xi \in V$ the differential $D_{v_{0}} S(t, w) \xi$ solves (3.12).

Before we give the contraction of sufficiently high dimensional volumes, the following two lemma is crucial.

LEMMA 3.3. For every family of elements $U_{1}, \cdots, U_{d}$ of $V$, we have

$$
\begin{aligned}
& \left(F^{\prime}(v(t)) U_{1} \wedge\left(I-\alpha^{2} \Delta\right) U_{2} \wedge \cdots\left(I-\alpha^{2} \Delta\right) U_{d}, U_{1} \wedge \cdots U_{d}\right)_{\wedge^{d} H}+\cdots \\
& \quad+\left(\left(I-\alpha^{2} \Delta\right) U_{1} \wedge\left(I-\alpha^{2} \Delta\right) U_{2} \wedge \cdots F^{\prime}(v(t)) U_{d}, U_{1} \wedge \cdots U_{d}\right)_{\wedge^{d} H} \\
& =\left|U_{1} \wedge \cdots \wedge U_{d}\right|_{\wedge^{d} V}^{2} \sum_{j=1}^{d}\left\langle F^{\prime}(v(t)) \vartheta_{j}, \vartheta_{j}\right\rangle_{H},
\end{aligned}
$$

where $\vartheta_{j} j=1, \cdots, d$, be an orthonormal basis of $\operatorname{Span}\left[U_{1}, \cdots, U_{d}\right]$ in $V$.

Proof. As in [22], we consider the case where $U_{1}, \cdots, U_{d}$ are linearly independent, $U_{1} \wedge \cdots U_{d} \neq 0$ and, without loss of generality, we can assume that $U_{1}, \cdots, U_{d}$ is an orthogonal family of $V$. If $U_{1}, \cdots, U_{d}$ is not orthogonal we can consider the orthogonal family $\psi_{1}, \cdots, \psi_{d}$ associated with $U_{1}, \cdots, U_{d}$, by the usual Gram-Schmidt process. Since

$$
\psi_{j} \in U_{j}+\operatorname{Span}\left\{U_{1}, U_{j-1}\right\}, \quad j=1, \cdots, d,
$$

we have clear

$$
\psi_{1} \wedge \cdots \wedge \psi_{d}=U_{1} \wedge \cdots \wedge U_{d}
$$


and

$$
\left|\psi_{1} \wedge \cdots \wedge \psi_{d}\right|_{\wedge^{d} V}^{2}=\left\|\psi_{1}\right\|_{V}^{2} \cdots\left\|\psi_{d}\right\|_{V}^{2}
$$

Furthermore, since linearity of $F^{\prime}(v(t))$ and $I-\alpha^{2} \Delta$, by the fact that $\psi_{1}, \cdots, \psi_{d}$ is the orthogonal family in $V$ and (3.16), we obtain

$$
\begin{aligned}
\left(F^{\prime}(v(t)) U_{1} \wedge\left(I-\alpha^{2} \Delta\right) U_{2} \wedge \cdots\left(I-\alpha^{2} \Delta\right) U_{d}, U_{1} \wedge \cdots U_{d}\right)_{\wedge^{d} H}+\cdots \\
\quad+\left(\left(I-\alpha^{2} \Delta\right) U_{1} \wedge\left(I-\alpha^{2} \Delta\right) U_{2} \wedge \cdots F^{\prime}(v(t)) U_{d}, U_{1} \wedge \cdots U_{d}\right)_{\wedge^{d} H} \\
=\left(F^{\prime}(v(t)) \psi_{1} \wedge\left(I-\alpha^{2} \Delta\right) \psi_{2} \wedge \cdots\left(I-\alpha^{2} \Delta\right) \psi_{d}, \psi_{1} \wedge \cdots \psi_{d}\right)_{\wedge^{d} H}+\cdots \\
\quad+\left(\left(I-\alpha^{2} \Delta\right) \psi_{1} \wedge\left(I-\alpha^{2} \Delta\right) \psi_{2} \wedge \cdots F^{\prime}(v(t)) \psi_{d}, \psi_{1} \wedge \cdots \psi_{d}\right)_{\wedge^{d} H} \\
=\left\langle F^{\prime}(v(t)) \psi_{1}, \psi_{1}\right\rangle_{H}\left\|\psi_{2}\right\|_{V}^{2} \cdots\left\|\psi_{d}\right\|_{V}^{2}+\cdots \\
+\left\|\psi_{1}\right\|_{V}^{2} \cdots\left\|\psi_{d-1}\right\|_{V}^{2}\left\langle F^{\prime}(v(t)) \psi_{d}, \psi_{d}\right\rangle_{H} \\
=\left\|\psi_{1}\right\|_{V}^{2} \cdots\left\|\psi_{d}\right\|_{V}^{2} \sum_{j=1}^{d}\left\langle\frac{F^{\prime}(v(t)) \psi_{j}}{\left\|\psi_{j}\right\|_{V}}, \frac{\psi_{j}}{\left\|\psi_{j}\right\|_{V}}\right\rangle_{H} \\
=\left|\psi_{1} \wedge \cdots \wedge \psi_{d}\right|_{\wedge^{d} V}^{2} \sum_{j=1}^{d}\left\langle\frac{F^{\prime}(v(t)) \psi_{j}}{\left\|\psi_{j}\right\|_{V}}, \frac{\psi_{j}}{\left\|\psi_{j}\right\|_{V}}\right\rangle_{H} \\
=\left|U_{1} \wedge \cdots \wedge U_{d}\right|_{\wedge^{d} V}^{2} \sum_{j=1}^{d}\left\langle\frac{F^{\prime}(v(t)) \psi_{j}}{\left\|\psi_{j}\right\|_{V}}, \frac{\psi_{j}}{\left\|\psi_{j}\right\|_{V}}\right\rangle_{H} \\
=\left|U_{1} \wedge \cdots \wedge U_{d}\right|_{\wedge^{d} V}^{2} \sum_{j=1}^{d}\left\langle F^{\prime}(v(t)) \vartheta_{j}, \vartheta_{j}\right\rangle_{H} .
\end{aligned}
$$

Lemma 3.4. Suppose $\xi_{1}, \cdots, \xi_{d} \in V$, and denote $D_{v_{0}} S(t, w) \xi_{k}$ by $U_{k}, k=$ $1, \cdots, d$. Then $U_{k}$ solves $(3.12)-(3.13), k=1, \cdots, d$, and

$$
\begin{aligned}
& \left|U_{1}(t) \wedge \cdots \wedge U_{d}(t)\right|_{\wedge^{d} V}^{2} \\
& \quad=\left|\xi_{1} \wedge \cdots \wedge \xi_{d}\right|_{\wedge^{d} V}^{2} \exp \left(\int_{0}^{t} \sum_{j=1}^{d}\left\langle F^{\prime}(v(\tau)) \vartheta_{j}, \vartheta_{j}\right\rangle_{H} d \tau\right) .
\end{aligned}
$$

Proof. We rewrite the norm

$$
\|\phi\|_{V}^{2}=\|\phi\|^{2}+\alpha^{2}\|\phi\|_{1}^{2}
$$

for all $\phi \in V$, thanks to the Poincaré inequality, we have this norm is equivalent to the former norm $\|\cdot\|_{1}$. 
By time differentiation, applying (3.19) and Lemma 3.3, we obtain

$$
\begin{aligned}
& \frac{1}{2} \frac{d}{d t}\left|U_{1}(t) \wedge \cdots \wedge U_{d}(t)\right|_{\wedge^{d} V}^{2} \\
& =\left(\frac{d}{d t}\left(U_{1}(t) \wedge \cdots \wedge U_{d}(t)\right), U_{1}(t) \wedge \cdots \wedge U_{d}(t)\right)_{\wedge^{d} V} \\
& \left.=\left(U_{1}^{\prime}(t) \wedge \cdots \wedge U_{d}(t)\right), U_{1}(t) \wedge \cdots \wedge U_{d}(t)\right)_{\wedge}{ }^{d} V \\
& \left.+\cdots+\left(U_{1} \wedge \cdots \wedge U_{d}^{\prime}(t)\right), U_{1}(t) \wedge \cdots \wedge U_{d}(t)\right)_{\wedge^{d} V} \\
& =\left|\begin{array}{cccc}
\left\langle U_{1}^{\prime}, U_{1}\right\rangle_{V} & \left\langle U_{1}^{\prime}, U_{2}\right\rangle_{V} & \cdots & \left\langle U_{1}^{\prime}, U_{d}\right\rangle_{V} \\
\left\langle U_{2}, U_{1}\right\rangle_{V} & \left\langle U_{2}, U_{2}\right\rangle_{V} & \cdots & \left\langle U_{2}, U_{d}\right\rangle_{V} \\
\cdots & \cdots & \cdots & \cdots \\
\left\langle U_{d}, U_{1}\right\rangle_{V} & \left\langle U_{d}, U_{2}\right\rangle_{V} & \cdots & \left\langle U_{d}, U_{d}\right\rangle_{V}
\end{array}\right|+\cdots \\
& +\left|\begin{array}{cccc}
\left\langle U_{1}, U_{1}\right\rangle_{V} & \left\langle U_{1}, U_{2}\right\rangle_{V} & \cdots & \left\langle U_{1}, U_{d}\right\rangle_{V} \\
\left\langle U_{2}, U_{1}\right\rangle_{V} & \left\langle U_{2}, U_{2}\right\rangle_{V} & \cdots & \left\langle U_{2}, U_{d}\right\rangle_{V} \\
\cdots & \cdots & \cdots & \cdots \\
\left\langle U_{d}^{\prime}, U_{1}\right\rangle_{V} & \left\langle U_{d}^{\prime}, U_{2}\right\rangle_{V} & \cdots & \left\langle U_{d}^{\prime}, U_{d}\right\rangle_{V}
\end{array}\right| \\
& =\left|\begin{array}{cccc}
\left\langle\left(I-\alpha^{2} \Delta\right) U_{1}^{\prime}, U_{1}\right\rangle_{H} & \left\langle\left(I-\alpha^{2} \Delta\right) U_{1}^{\prime}, U_{2}\right\rangle_{H} & \cdots & \left\langle\left(I-\alpha^{2} \Delta\right) U_{1}^{\prime}, U_{d}\right\rangle_{H} \\
\left\langle\left(I-\alpha^{2} \Delta\right) U_{2}, U_{1}\right\rangle_{H} & \left\langle\left(I-\alpha^{2} \Delta\right) U_{2}, U_{2}\right\rangle_{H} & \cdots & \left\langle\left(I-\alpha^{2} \Delta\right) U_{2}, U_{d}\right\rangle_{H} \\
\cdots & \cdots & \cdots & \cdots \\
\left\langle\left(I-\alpha^{2} \Delta\right) U_{d}, U_{1}\right\rangle_{H} & \left\langle\left(I-\alpha^{2} \Delta\right) U_{d}, U_{2}\right\rangle_{H} & \cdots & \left\langle\left(I-\alpha^{2} \Delta\right) U_{d}, U_{d}\right\rangle_{H}
\end{array}\right|+\cdots \\
& +\left|\begin{array}{cccc}
\left\langle\left(I-\alpha^{2} \Delta\right) U_{1}, U_{1}\right\rangle_{H} & \left\langle\left(I-\alpha^{2} \Delta\right) U_{1}, U_{2}\right\rangle_{H} & \cdots & \left\langle\left(I-\alpha^{2} \Delta\right) U_{1}, U_{d}\right\rangle_{H} \\
\left\langle\left(I-\alpha^{2} \Delta\right) U_{2}, U_{1}\right\rangle_{H} & \left\langle\left(I-\alpha^{2} \Delta\right) U_{2}, U_{2}\right\rangle_{H} & \cdots & \left\langle\left(I-\alpha^{2} \Delta\right) U_{2}, U_{d}\right\rangle_{H} \\
\cdots & \cdots & \cdots & \cdots \\
\left\langle\left(I-\alpha^{2} \Delta\right) U_{d}^{\prime}, U_{1}\right\rangle_{H} & \left\langle\left(I-\alpha^{2} \Delta\right) U_{d}^{\prime}, U_{2}\right\rangle_{H} & \cdots & \left\langle\left(I-\alpha^{2} \Delta\right) U_{d}^{\prime}, U_{d}\right\rangle_{H}
\end{array}\right| \\
& =\left(F^{\prime}(v(t)) U_{1} \wedge\left(I-\alpha^{2} \Delta\right) U_{2} \wedge \cdots\left(I-\alpha^{2} \Delta\right) U_{d}, U_{1} \wedge \cdots U_{d}\right)_{\wedge^{d} H}+\cdots \\
& +\left(\left(I-\alpha^{2} \Delta\right) U_{1} \wedge\left(I-\alpha^{2} \Delta\right) U_{2} \wedge \cdots F^{\prime}(v(t)) U_{d}, U_{1} \wedge \cdots U_{d}\right)_{\wedge^{d} H}
\end{aligned}
$$

$$
=\left|U_{1} \wedge \cdots \wedge U_{d}\right|_{\wedge^{d} V}^{2} \sum_{j=1}^{d}\left\langle F^{\prime}(v(t)) \vartheta_{j}, \vartheta_{j}\right\rangle_{H} .
$$

Therefore

$$
\begin{aligned}
& \left|U_{1}(t) \wedge \cdots \wedge U_{d}(t)\right|_{\wedge^{d} V} \\
& \quad=\left|\xi_{1} \wedge \cdots \wedge \xi_{d}\right|_{\wedge^{d} V} \exp \left(\int_{0}^{t} \sum_{j=1}^{d}\left\langle F^{\prime}(v(\tau)) \vartheta_{j}, \vartheta_{j}\right\rangle_{H} d \tau\right) .
\end{aligned}
$$

REMARK 3.5. It is worth stressing that since adding the regularizing term $\left(-\alpha \Delta v_{t}\right)$ to the Navier Stokes equations, the linear initial-value problem (3.12)(3.13) is different to the one in Section V.2.3 in $[\mathbf{2 2}]$, in which for every $d \in \mathbb{N}$,

$$
\begin{aligned}
& \left|U_{1}(t) \wedge \cdots \wedge U_{d}(t)\right|_{\wedge^{d} V}^{2} \\
& \quad=\left|\xi_{1} \wedge \cdots \wedge \xi_{d}\right|_{\wedge^{d} V}^{2} \exp \left(\int_{0}^{t} \operatorname{Tr} F^{\prime}\left(S(\tau) \chi_{0}\right) \circ Q_{d}(\tau) d \tau\right),
\end{aligned}
$$


where $S(\tau) \chi_{0}=\chi(\tau)=(v(\tau), \theta(\tau))$ and $Q_{d}$ is the orthogonal projector in $V$ onto the space spanned by $U_{1}(\tau), \ldots, U_{d}(\tau)$. However, applying Lemma 3.3 and Lemma 3.4, we have (3.18) here.

Volume contraction is shown as follows.

TheOREM 3.6. Let $S$ be the flow associated with (3.10) and let $w \mapsto \mathcal{A}(w)$ be the global attractor given by Theorem 3.2, then there exist a positive integer $d$ and a time $t>0$, such that

$$
\left\|\Gamma_{d}(t, \cdot)\right\|_{L^{\infty}}<1
$$

where $\Gamma_{d}(t, \cdot)=\sup _{v \in \mathcal{A}(w)}\left\|\wedge^{d} D_{v} S(t, w)\right\|$.

Proof. Suppose $\xi_{1}, \cdots, \xi_{d} \in V, v_{0} \in V$, and denote $D_{v_{0}} S(t, w) \xi_{k}$ by $U_{k}, k=$ $1, \cdots, d$. Then according to Lemma 3.4, we have

$$
\begin{aligned}
& \left|U_{1}(t) \wedge \cdots \wedge U_{d}(t)\right|_{\wedge^{d} V}^{2} \\
& \quad=\left|\xi_{1} \wedge \cdots \wedge \xi_{d}\right|_{\wedge^{d} V}^{2} \exp \left(\int_{0}^{t} \sum_{j=1}^{d}\left\langle F^{\prime}(v(\tau)) \vartheta_{j}, \vartheta_{j}\right\rangle_{H} d \tau\right) .
\end{aligned}
$$

By the Gagliardo-Nirenberg inequality, Poincaré inequality and the Young's inequality, we deduce

$$
\begin{aligned}
& \sum_{j=1}^{d}\left\langle F^{\prime}(v(t)) \vartheta_{j}, \vartheta_{j}\right\rangle_{H} \\
& =\sum_{j=1}^{d}\left\langle-\nu A \vartheta_{j}+B\left(S(t, w) v_{0}, \vartheta_{j}\right)+B\left(\vartheta_{j}, S(t, w) v_{0}\right), \vartheta_{j}\right\rangle_{H} \\
& \leq-\nu \sum_{j=1}^{d}\left\|\vartheta_{j}\right\|_{1}^{2}+\left|\sum_{j=1}^{d} b\left(\vartheta_{j}, v, \vartheta_{j}\right)\right| \\
& \leq-\nu \sum_{j=1}^{d}\left\|\vartheta_{j}\right\|_{1}^{2}+\sum_{j=1}^{d}\left\|\vartheta_{j}\right\|_{L^{4}}^{2}\|v\|_{1} \\
& \leq-\nu \sum_{j=1}^{d}\left\|\vartheta_{j}\right\|_{1}^{2}+\|v\|_{1} \sum_{j=1}^{d}\left\|\vartheta_{j}\right\|_{1}^{\frac{3}{2}}\left\|\vartheta_{j}\right\|^{\frac{1}{2}} \\
& \leq-\nu d+\|v\|_{1} \sum_{j=1}^{d}\left\|\vartheta_{j}\right\|^{\frac{1}{2}} \\
& \leq-\nu d+\frac{\nu}{2} \sum_{j=1}^{d}\left\|\vartheta_{j}\right\|_{1}+c\|v\|_{1}^{2} \\
& \leq-\frac{\nu}{2} d+c\|v\|_{1}^{2} .
\end{aligned}
$$

Therefore

$$
\begin{aligned}
& \left|U_{1}(t) \wedge \cdots \wedge U_{d}(t)\right|_{\wedge^{d} V}^{2} \\
& \quad \leq\left|\xi_{1} \wedge \cdots \wedge \xi_{d}\right|_{\wedge^{d} V}^{2} \exp \left(-\frac{\nu d t}{2}+c \int_{0}^{t}\|v(\tau)\|_{1}^{2} d \tau\right),
\end{aligned}
$$


for every fixed $t>0$.

Since $v(\tau)=S(\tau, w) v_{0} \in \mathcal{A}\left(\theta_{\tau} w\right)$ and Theorem 3.2, moreover, by ergodicity of the shift $\theta_{\tau}$, we obtain, for $\mathbb{P}$-a.s.,

$$
\begin{aligned}
\lim _{t \rightarrow \infty} \frac{1}{t} \int_{0}^{t}\|v(\tau)\|_{1}^{2} d \tau & \leq \lim _{t \rightarrow \infty} \frac{1}{t} \int_{0}^{t} R_{0}\left(\theta_{\tau} w\right)^{2} d \tau \\
& \leq \mathbb{E} R_{0}(w)^{2}
\end{aligned}
$$

then for $\forall t>0$, we have $\int_{0}^{t}\|v(\tau)\|_{1}^{2} d \tau \leq c_{1}(w)+c_{2}(w) t$, where the constant $c_{1}$ and $c_{2}$ are random and finite $\mathbb{P}-$ a.s..

Recalling the notations for $\Gamma_{d}(t, \cdot)$, we get

$$
\Gamma_{d}(t, w) \leq e^{\left(-\frac{\nu d}{2}+c_{2}\right) t+c_{1}}, \quad \mathbb{P}-a . s .
$$

for every $t>0$, let $d$ sufficiently large, we obtain $\left\|\Gamma_{d}(t, \cdot)\right\|_{L^{\infty}}<1$ for $\mathbb{P}$-a.s..

\subsection{Finite Dimension of the Attractor.}

THEOREM 3.7. The Hausdorff dimension of the global attractor for the SNSV is finite.

Proof. From Theorem 3.6 we get existence of some $d$ such that

$$
\left\|\Gamma_{d}(t, \cdot)\right\|_{L^{\infty}}<1 .
$$

This is the first basic estimate we need to apply Theorem 2.2.

From the proof of Theorem 3.6, we get for the particular case $d=1$

$$
\left\|U_{1}\right\|_{V} \leq\left\|\xi_{1}\right\|_{V} e^{c_{1}+c_{2} t} .
$$

Thus the assumption $\gamma_{1}(t) \in L^{\infty}(\Omega, \mathbb{P})$ is fulfilled. Finally, we have to prove that $g_{\delta}(t)$ convergence to 0 in $L^{\infty}(\Omega, \mathbb{P})$ for each $t>0$.

Let $x \in V$ and $x_{0} \in \mathcal{A}(w)$ be given, and put $u(t)=S(t, w) x, v(t)=S(t, w) x_{0}$, $z(t)=D_{x_{0}} S(t, w)\left(x-x_{0}\right)$ and $\eta(t)=u(t)-v(t)-z(t)$. Then

$$
(u-v)_{t}+\alpha^{2} A(u-v)_{t}=-\nu A(u-v)-B(u, u)+B(v, v)
$$

and

$$
z_{t}+\alpha^{2} z_{t}=-\nu A z_{t}-B(v, z)-B(z, v)
$$

hence

$$
\eta_{t}+\alpha^{2} \eta_{t}=-\nu A \eta-B(v, \eta)-B(\eta, v)-B(u-v, u-v) .
$$

Therefore

$$
\begin{aligned}
\frac{1}{2} \frac{d}{d t}\left(\|\eta\|^{2}+\alpha^{2}\|\eta\|_{1}^{2}\right) & \leq-\nu\|\eta\|_{1}^{2}+\langle B(\eta, v), \eta\rangle_{H}+\langle B(u-v, u-v), \eta\rangle_{H} \\
& \leq-\nu\|\eta\|_{1}^{2}+c_{3}\|\eta\|_{1}^{2}\|v\|_{1}+c_{4}\|u-v\|^{\frac{1}{2}}\|u-v\|_{1}^{\frac{3}{2}}\|\eta\|_{1} \\
& \leq-\frac{\nu}{2}\|\eta\|_{1}^{2}+\frac{c_{3}}{\alpha^{2}}\|v\|_{1}\left(\|\eta\|^{2}+\alpha^{2}\|\eta\|_{1}^{2}\right)+\frac{c_{4}}{2 \nu}\|u-v\|\|u-v\|_{1}^{3} .
\end{aligned}
$$

By the Gronwall lemma, for $\mathbb{P}$-a.s., we get

$$
\begin{aligned}
\|\eta\|^{2}+\alpha^{2}\|\eta\|_{1}^{2} & \leq \frac{c_{4}}{\nu} \int_{0}^{t} e^{\int_{s}^{t} \frac{2 c_{3}}{\alpha^{2}}\|v(\sigma)\|_{1} d \sigma}\|u-v\|\|u-v\|_{1}^{3} d s \\
& \leq \frac{c_{4}}{\nu} e^{\frac{2 c_{3}}{\alpha^{2}}\left(c_{1}+c_{2} t\right)} \int_{0}^{t}\|u-v\|\|u-v\|_{1}^{3} d s
\end{aligned}
$$


Taking the inner product of (3.28) with $u-v$, one has

$$
\begin{aligned}
\frac{1}{2} \frac{d}{d t}\left(\|u-v\|^{2}+\alpha^{2}\|u-v\|_{1}^{2}\right) & \leq-\nu\|u-v\|_{1}^{2}-\langle B(u-v, v), u-v\rangle_{H} \\
& \leq \frac{c_{5}}{\alpha^{2}}\left(\|u-v\|^{2}+\alpha^{2}\|u-v\|_{1}^{2}\right)\|v\|_{1},
\end{aligned}
$$

then we have

$$
\begin{aligned}
\|u-v\|^{2}+\alpha^{2}\|u-v\|_{1}^{2} & \leq e^{\frac{2 c_{5}}{\alpha^{2}} \int_{0}^{t}\|v\|_{1} d s}\left(\left\|x-x_{0}\right\|^{2}+\alpha^{2}\left\|x-x_{0}\right\|_{1}^{2}\right) \\
& \leq e^{\frac{2 c_{5}}{\alpha^{2}}\left(c_{1}+c_{2} t\right)}\left\|x-x_{0}\right\|_{1}^{2}
\end{aligned}
$$

By (3.29)-(3.30), one has

$$
\|\eta\|_{1}^{2} \leq c(t)\left\|x-x_{0}\right\|_{1}^{4}
$$

where $c(t)>0$ depending on $c_{1}, c_{2}, c_{3}, c_{4}, c_{5}, \alpha, t$, for $\mathbb{P}$-a.s.. Recalling the definition of $g_{\delta}(t)$, we see that it converges to 0 in $L^{\infty}(\Omega, \mathbb{P})$ for each $t>0$. Thus Theorem 2.2 applies.

\section{Finite Dimension of the Attractor for SPEs}

4.1. Existence of Attractor. Given a smooth bounded domain $M \subset \mathbb{R}^{2}$ and the cylindrical domain $\mho=M \times(-h, 0) \subset \mathbb{R}^{3}$, our aim is to study the following $3 \mathrm{D}$ viscous SPEs with additive noise in $\mho$.

$$
(S P E s)\left\{\begin{array}{l}
\frac{\partial v}{\partial t}+(v \cdot \nabla) v+\eta \frac{\partial v}{\partial z}+\nabla p+f v^{\perp}+L_{1} v=\sum_{j=1}^{m} \phi_{j} \frac{d w_{j}(t)}{d t} \\
\partial_{z} p+\theta=0 \\
\nabla \cdot v+\partial_{z} \eta=0 \\
\frac{\partial \theta}{\partial t}+v \cdot \nabla \theta+\eta \frac{\partial \theta}{\partial z}+L_{2} \theta=Q
\end{array}\right.
$$

with fluid velocity field $(u, w)=\left(u_{1}, u_{2}, w\right) \in \mathbb{R}^{3}$ with $u=\left(u_{1}, u_{2}\right)$ and $u^{\perp}=$ $\left(-u_{2}, u_{1}\right)$ being horizontal, the temperature $\theta, \phi=(u, \theta)$ and the pressure $p ; f=$ $f_{0}(\beta+y)$ is the given Coriolis rotation frequency with $\beta$-plane approximation and $Q$ is a given heat source. The viscosity and the heat diffusion operators $L_{1}$ and $L_{2}$ are given respectively as the following:

$$
L_{i}=-\nu_{i} \Delta-\mu_{i} \frac{\partial^{2}}{\partial z^{2}}, \quad i=1,2
$$

here the positive constants $\nu_{1}, \mu_{1}$ are the horizontal and vertical viscous coefficients; while the positive constants $\nu_{2}, \mu_{2}$ are the horizontal and vertical heat diffusivity coefficients, without lost of generality in this paper, we take $\nu_{1,2}, \mu_{1,2}$ to be 1 . In the above equations and in this article, we denote by $\nabla$ the horizontal gradient operator and by $\Delta$ the horizontal Laplacian:

$$
\nabla \equiv\left(\partial_{x}, \partial_{y}\right), \quad \Delta \equiv \partial_{x}^{2}+\partial_{y}^{2}
$$

and

$$
\nabla_{3} \equiv\left(\partial_{x}, \partial_{y}, \partial_{z}\right), \quad \Delta_{3} \equiv \partial_{x}^{2}+\partial_{y}^{2}+\partial_{z}^{2}
$$

The function $\phi_{j}=\phi_{j}(x, y), j=1, \ldots, m$, are time independent and specified below. The random function $w_{j}, j=1, \ldots, m$, are independent two-sided realvalue Wiener processes on a probability space $(\Omega, \mathscr{F}, \mathbb{P})$. More precisely, let $\Omega=\left\{\omega \in C\left(\mathbb{R}, \mathbb{R}^{m}\right) \mid\right.$ 
$\omega(0)=0\}$, with $\mathbb{P}$ being a product measure of two Wiener measure on the negative and the positive time parts of $\Omega$; then we have

$$
\left(w_{1}(t, \omega), w_{2}(t, \omega), \ldots, w_{m}(t, \omega)\right)=\omega(t), \quad t \in \mathbb{R},
$$

the time shift is simply defined by

$$
\vartheta_{s} \omega(t)=\omega(t+s)-\omega(s), \quad t, s \in \mathbb{R},
$$

it is a family or ergodic transformations.

The boundary of $\mho$ is partitioned into three parts: $\partial \mho=\Gamma_{u} \cup \Gamma_{b} \cup \Gamma_{s}$, where

$$
\begin{aligned}
& \Gamma_{u}=\{(x, y, z) \in \bar{\mho}: z=0\}, \\
& \Gamma_{b}=\{(x, y, z) \in \bar{\mho}: z=-h\}, \\
& \Gamma_{s}=\{(x, y, z) \in \bar{\mho}:(x, y) \in \partial M,-h \leq z \leq 0\},
\end{aligned}
$$

here $h>0$ is a given constant. We consider the following boundary conditions:

$$
\begin{aligned}
& \Gamma_{u}: \frac{\partial v}{\partial z}=0, \quad w=0, \quad \frac{\partial \theta}{\partial z}=0 \\
& \Gamma_{b}: \frac{\partial v}{\partial z}=0, \quad w=0, \quad \frac{\partial \theta}{\partial z}=0 \\
& \Gamma_{s}: v \cdot n=0, \quad \frac{\partial v}{\partial n} \times n=0, \quad \frac{\partial \theta}{\partial n}=0 .
\end{aligned}
$$

We define the following Hilbert spaces as in [11]:

$$
\begin{gathered}
H=H_{1} \times H_{2}, \quad V=V_{1} \times V_{2}, \\
H_{1}=\left\{v \in L_{2}(\mho)^{2}|\nabla \cdot \bar{v}=0, \quad v \cdot n| \Gamma_{s}=0\right\}, \quad H_{2}=L_{2}(\mho), \\
V_{1}=\left\{v \in H_{1}(\mho)^{2}|\nabla \cdot \bar{v}=0, \quad v \cdot n| \Gamma_{s}=0\right\}, \quad V_{2}=H_{1}(\mho) .
\end{gathered}
$$

These spaces are endowed with the $L_{2}$ and $H_{1}$ norms which we respectively denote by $|\cdot|_{2}$ and $\|\cdot\|$. Let $V^{\prime}$ be the dual space of $V$. We have the dense and continuous embeddings $V \hookrightarrow H=H^{\prime} \hookrightarrow V^{\prime}$ and denote by $\langle\phi, \psi\rangle$ the duality between $\phi \in V$ (resp. $V_{i}$ ) and $\phi \in V^{\prime}$ (resp. $V_{i}^{\prime}$ ).

Consider an unbounded linear operator $A=\left(L_{1}, L_{2}\right): H \rightarrow H$ with $D(A)=$ $D\left(L_{1}\right) \times D\left(L_{2}\right)$ where $D\left(L_{1}\right)=V_{1} \cap H^{2}(\mho), D\left(L_{2}\right)=V_{2} \cap H^{2}(\mho)$ and define

$$
\left\langle L_{1} u, v\right\rangle=((u, v)),\left\langle L_{2} \theta, \eta\right\rangle=((\theta, \eta)), \forall u, v \in D\left(L_{1}\right), \forall \theta, \eta \in D\left(L_{2}\right) .
$$

The operator $L_{1}$ and $L_{2}$ are self-adjoint, positive, with compact self-adjoint inverses. They map $V$ to $V^{\prime}$.

Let $\delta>0$ be given, we shall impose condition below on $\delta$. For each $k=1, \ldots, m$, let $\varsigma_{k}$ be the stationary solution of the Ornstein-Uhlenbeck process

$$
d \varsigma_{k}=-\delta \varsigma_{k} d t+d w_{k}(t)
$$

So

$$
\varsigma_{k}(t)=\int_{-\infty}^{t} e^{-\delta(t-s)} d w_{k}(s) .
$$

Putting $\varsigma(t)=\sum_{k=1}^{m} \phi_{k} \varsigma_{k}(t)$ we have

$$
d \varsigma=-\delta \varsigma d t+d w(t)
$$


where $w(t)=\sum_{k=1}^{m} \phi_{k} w_{k}(t)$. Since the trajectories of $\varsigma_{k}$ are $\mathbb{P}$-a.s. continuous and $\phi_{k} \in D\left(A_{1}\right)$, we have

$$
\sup _{-n \leq t \leq n}\left\{\|\varsigma(t)\|_{H_{1}}^{2}+\|\varsigma(t)\|_{V_{1}}^{2}+\left\|A_{1} \varsigma(t)\right\|_{H_{1}}^{2}\right\} \leq M, \quad \mathbb{P}-\text { a.s. }
$$

Letting $u(t)=v(t)-\varsigma(t)$, then $u$ satisfies the equations (which depends on a random parameter):

(4.8) $\left.\quad \frac{\partial u}{\partial z}\right|_{\Gamma_{u}}=\left.\frac{\partial u}{\partial z}\right|_{\Gamma_{b}}=0,\left.\quad u \cdot n\right|_{\Gamma_{s}}=0, \quad \frac{\partial u}{\partial n} \times\left. n\right|_{\Gamma_{s}}=0$

(4.9) $\left.\quad\left(\frac{\partial \theta}{\partial z}+\alpha \theta\right)\right|_{\Gamma_{u}}=\left.\frac{\partial \theta}{\partial z}\right|_{\Gamma_{b}}=0,\left.\quad \frac{\partial \theta}{\partial n}\right|_{\Gamma_{s}}=0$,

(4.10) $u\left(x, y, z, t_{0}, \omega\right)=v_{0}(x, y, z, \omega)-\varsigma_{0}(x, y, \omega), \theta\left(x, y, z, t_{0}, \omega\right)=\theta_{0}(x, y, z, \omega)$.

In $[\mathbf{1 1}]$, we have already obtained the following wellposedness for the problems (4.6)-(4.10).

TheOREM 4.1. Assume that $t_{0} \in \mathbb{R}$ and $\left(u_{0}, \theta_{0}\right) \in V_{1} \times V_{2}$, for $\mathbb{P}$-almost every $\omega \in \Omega$ the following holds:

(i) there exist a unique strong solution of (4.6)-(4.10) on the time interval $\left[t_{0}, \infty\right)$ and satisfies $u \in C\left(\left[t_{0}, \infty\right), V_{1}\right) \cap L^{2}\left(t_{0}, \infty ; D\left(A_{1}\right)\right), \theta \in C\left(\left[t_{0}, \infty\right), V_{2}\right) \cap$ $L^{2}\left(t_{0}, \infty ; D\left(A_{2}\right)\right)$.

(ii) denoting such solution by $u\left(t, \omega ; t_{0}, u_{0}\right)$, the mapping $u_{0} \mapsto u\left(t, \omega ; t_{0}, u_{0}\right)$ is continuous for all $t \geq t_{0}$.

In [11], by uniform estimates and Aubin-Lions compactness lemma combined with the use of the Riesz lemma and a continuity argument, we showed that the long time dynamics is captured by a random attractor. V-weak topology attractor was given in $[\mathbf{1 3}]$.

THEOREM 4.2. (see $[\mathbf{1 1}]$ ) Let $K(w)$ be the ball in $V$ of radius $R_{0}(w)$ such that for any $B$ bounded in $V=V_{1} \times V_{2}$, there exists $s_{1}(w, B)$, for $s<s_{1}(w, B)$, we have

$$
S(0, s ; w) B \subset K(w), \quad \mathbb{P}-\text { a.s. }
$$

and the stochastic flow $S$ is asymptotically compact in $V$ for $\mathbb{P}$-a.s., thus we obtain a random attractor $\mathcal{A}(\omega)$ which attracts every bounded nonrandom set $B \subset V$. 
4.2. Volume Contraction for SPEs. Consider the differential equation on $V=V_{1} \times V_{2}$, here $U$ will denote a pair $(\varrho, \psi)$.

$$
\begin{aligned}
& \frac{\partial \varrho}{\partial t}+L_{1} \varrho+(\varrho \cdot \nabla) v+(v \cdot \nabla) \varrho-\left(\int_{-h}^{z} \nabla \cdot \varrho(x, y, \xi, t) d \xi\right) \frac{\partial v}{\partial z} \\
& -\left(\int_{-h}^{z} \nabla \cdot v(x, y, \xi, t) d \xi\right) \frac{\partial \varrho}{\partial z}-\int_{-h}^{z} \nabla \psi(x, y, \xi, t) d \xi+f \varrho^{\perp}=0, \\
& \frac{\partial \psi}{\partial t}+L_{2} \psi+v \cdot \nabla \psi+\varrho \cdot \nabla \theta-\left(\int_{-h}^{z} \nabla \cdot \varrho(x, y, \xi, t) d \xi\right) \frac{\partial \theta}{\partial z} \\
& \quad-\left(\int_{-h}^{z} \nabla \cdot v(x, y, \xi, t) d \xi\right) \frac{\partial \psi}{\partial z}=0, \\
& U(0, w)=(\varrho(0, w), \psi(0, w))=\xi \in V .
\end{aligned}
$$

The existence of a solution $U \in C\left(\left[t_{0}, \infty\right), V\right) \cap L^{2}\left(t_{0}, \infty ; D(A)\right)$ for all $T>0$, of (4.12)-(4.14) can be established, path by path, as in the deterministic case. For $\xi=\xi_{1}, \ldots, \xi_{d} \in V$, let $U_{1}, \ldots, U_{d}$ denote the corresponding solutions of (4.12)(4.14), hence following the procedure in Section V.2.3 in [22], we consider, for every $d \in \mathbb{N}$,

$$
\begin{aligned}
& \left|U_{1}(t) \wedge \cdots \wedge U_{d}(t)\right|_{\wedge^{d} V}^{2} \\
& \quad=\left|\xi_{1} \wedge \cdots \wedge \xi_{d}\right|_{\wedge^{d} V}^{2} \exp \left(\int_{0}^{t} \operatorname{Tr} F^{\prime}\left(S(\tau) \chi_{0}\right) \circ Q_{d}(\tau) d \tau\right),
\end{aligned}
$$

where $S(\tau) \chi_{0}=\chi(\tau)=(v(\tau), \theta(\tau))$ and $Q_{d}$ is the orthogonal projector in $V$ onto the space spanned by $U_{1}(\tau), \ldots, U_{d}(\tau)$.

At a given time $\tau$, let $\varphi_{j}(\tau)=\left(\varrho_{j}(\tau), \psi_{j}(\tau)\right), j \in \mathbb{N}$, be an orthonormal basis of $V$, hence the trace of $\operatorname{Tr} F^{\prime}\left(S(\tau) \chi_{0}\right) \circ Q_{d}(\tau)$ is given by

$$
\begin{aligned}
\operatorname{Tr} F^{\prime}\left(S(\tau) \chi_{0}\right) \circ Q_{d}(\tau) & \leq \sum_{j=1}^{d}-\left|L_{1} \varrho_{j}\right|^{2}-\left|L_{2} \psi_{j}\right|^{2}+\left|b_{1}\left(\varrho_{j}, v, \Delta_{3} \varrho_{j}\right)\right|+\left|b_{1}\left(v, \rho_{j}, \Delta_{3} \varrho_{j}\right)\right| \\
& +\left|\int_{\Omega}\left(\int_{-h}^{z} \nabla \cdot \varrho_{j}(x, y, \xi, t) d \xi\right) \frac{\partial v}{\partial z} \Delta_{3} \varrho_{j} d \Omega\right| \\
& +\left|\int_{\Omega}\left(\int_{-h}^{z} \nabla \cdot v(x, y, \xi, t) d \xi\right) \frac{\partial \varrho_{j}}{\partial z} \Delta_{3} \varrho_{j} d \Omega\right| \\
& +\left|\int_{\Omega}\left(\int_{-h}^{z} \nabla \psi_{j}(x, y, \xi, t) d \xi\right) \Delta_{3} \varrho_{j} d \Omega\right| \\
& +\left|b_{2}\left(\varrho_{j}, \theta, \Delta_{3} \psi_{j}\right)\right|+\left|b_{2}\left(v, \psi_{j}, \Delta_{3} \psi_{j}\right)\right| \\
& +\left|\int_{\Omega}\left(\int_{-h}^{z} \nabla \cdot \varrho_{j}(x, y, \xi, t) d \xi\right) \frac{\partial \theta}{\partial z} \Delta_{3} \psi_{j}\right| \\
& +\left|\int_{\Omega}\left(\int_{-h}^{z} \nabla \cdot v(x, y, \xi, t) d \xi\right) \frac{\partial \psi_{j}}{\partial z} \Delta_{3} \psi_{j}\right|,
\end{aligned}
$$


by the Hölder, Sobolev inequality and Lemma 4.2 in [14], we get

$$
\begin{aligned}
\operatorname{Tr} F^{\prime}\left(S(\tau) \chi_{0}\right) \circ Q_{d}(\tau) & \leq \sum_{j=1}^{d}\left(-\left|\Delta_{3} \varphi_{j}\right|_{L^{2}}^{2}+\left|\Delta_{3} \varrho_{j}\right|_{L^{2}}\|v\|_{V_{1}}^{\frac{1}{2}}\left|\Delta_{3} v\right|_{L^{2}}^{\frac{1}{2}}\left\|\varrho_{j}\right\|_{V_{1}}\right. \\
& +\left|\Delta_{3} \varrho_{j}\right|_{L^{2}}^{\frac{3}{2}}\left\|\varrho_{j}\right\|_{V_{1}}^{\frac{1}{2}}\|v\|_{V_{1}}+\left|\Delta_{3} \varrho_{j}\right|_{L^{2}}^{\frac{3}{2}}\left\|\varrho_{j}\right\|_{V_{1}}^{\frac{1}{2}}\left|v_{z}\right|_{L^{2}}^{\frac{1}{2}}\left|v_{z}\right|_{V_{1}}^{\frac{1}{2}} \\
& +\left|\Delta_{3} \varrho_{j}\right|_{L^{2}}^{\frac{3}{2}}\left\|\varrho_{j}\right\|_{V_{1}}^{\frac{1}{2}}|v|_{V_{1}}^{\frac{1}{2}}\left|\Delta_{3} v\right|_{L^{2}}^{\frac{1}{2}}+\left|\Delta_{3} \psi_{j}\right|_{L^{2}}\left\|\varrho_{j}\right\|_{V_{1}}|\theta|_{V_{2}}^{\frac{1}{2}}\left|\Delta_{3} \theta\right|_{L^{2}}^{\frac{1}{2}} \\
& +\left|\Delta_{3} \psi_{j}\right|_{L^{2}}^{\frac{3}{2}}\left\|\psi_{j}\right\|_{V_{1}}^{\frac{1}{2}}\|v\|_{V_{1}}+\left|\Delta_{3} \varphi_{j}\right|_{L^{2}}^{\frac{3}{2}}\left\|\varrho_{j}\right\|_{V_{1}}^{\frac{1}{2}}\left|\theta_{z}\right|_{L^{2}}^{\frac{1}{2}}\left|\theta_{z}\right|_{V_{2}}^{\frac{1}{2}} \\
& \left.+\left|\Delta_{3} \psi_{j}\right|_{L^{2}}^{\frac{3}{2}}\left\|\psi_{j}\right\|_{V_{1}}^{\frac{1}{2}}|v|_{V_{1}}^{\frac{1}{2}}\left|\Delta_{3} v\right|_{L^{2}}^{\frac{1}{2}}\right)
\end{aligned}
$$

Since $\left\{\varphi_{j}\right\}_{j}$ being orthonormal in $V=V_{1} \times V_{2}$, thus by Young's inequality, we have

$$
\begin{aligned}
\operatorname{Tr} F^{\prime}\left(S(\tau) \chi_{0}\right) \circ Q_{d}(\tau) \leq & \left(\sum_{j=1}^{d}-\frac{1}{2}\left|\Delta_{3} \varphi_{j}\right|_{L^{2}}^{2}\right)+|v|_{V_{1}}^{2}\left|\Delta_{3} v\right|_{L^{2}}^{2} \\
& +|\theta|_{V_{2}}^{2}\left|\Delta_{3} \theta\right|_{L^{2}}^{2}+\|v\|_{V_{1}}^{4}+1,
\end{aligned}
$$

applying Generalization of the Sobolev-Lieb-Thirring Inequality in $[\mathbf{2 2}]$, we have

$$
\sum_{j=1}^{d} \int_{\Omega}\left|\Delta_{3} \varphi_{j}\right|^{2} d \Omega \geq \frac{1}{\kappa_{2}}\left(\int_{\Omega}\left(\sum_{j=1}^{d}\left|\nabla \varphi_{j}\right|_{L^{2}}^{2}\right)^{2} d \Omega\right)^{\frac{2}{3}}-\frac{1}{|\Omega|^{\frac{2}{3}}} \int_{\Omega} \sum_{j=1}^{d}\left|\nabla \varphi_{j}\right|_{L^{2}}^{2} d \Omega
$$

$$
\geq d^{\frac{4}{3}}|\Omega|^{\frac{2}{3}} \frac{1}{\kappa_{2}}-d|\Omega|^{\frac{1}{3}} .
$$

We deduce that

$$
\begin{aligned}
& \left|U_{1}(t) \wedge \cdots \wedge U_{d}(t)\right|_{\wedge^{d} V}^{2} \\
& \quad \leq\left|\xi_{1} \wedge \cdots \wedge \xi_{d}\right|_{\wedge^{d} V}^{2} \exp \left(-d^{\frac{4}{3}}|\Omega|^{\frac{2}{3}} \frac{1}{2 \kappa_{2}} t+d\left(|\Omega|^{\frac{1}{3}}+1\right) t\right. \\
& \left.\quad+\int_{0}^{t}|v|_{V_{1}}^{2}\left|\Delta_{3} v\right|_{L^{2}}^{2}+|\theta|_{V_{2}}^{2}\left|\Delta_{3} \theta\right|_{L^{2}}^{2}+\|v\|_{V_{1}}^{4} d \tau\right) .
\end{aligned}
$$

Then we left the estimates of $\int_{0}^{t}|v|_{V_{1}}^{2}\left|\Delta_{3} v\right|_{L^{2}}^{2}+|\theta|_{V_{2}}^{2}\left|\Delta_{3} \theta\right|_{L^{2}}^{2}+\|v\|_{V_{1}}^{4} d \tau$.

Lemma 4.3. For any $\chi_{0}=\left(v_{0}, \theta_{0}\right) \in \mathcal{A}(w)$, we have $\chi(t)=S(t, w) \chi_{0}=$ $(v(t), \theta(t))$ satisfy

$$
\int_{0}^{t}|v|_{V_{1}}^{2}\left|\Delta_{3} v\right|_{L^{2}}^{2}+|\theta|_{V_{2}}^{2}\left|\Delta_{3} \theta\right|_{L^{2}}^{2}+\|v\|_{V_{1}}^{4} d \tau \leq C_{6}+C_{7} t
$$

for $\mathbb{P}$-a.s.

Proof. In [11], we get

$$
\begin{gathered}
\|\theta(t)\|_{2} \leq e^{-c t}\left\|\theta_{0}\right\|_{2}+\frac{1}{c}\|Q\|_{2}, \\
\int_{t}^{t+1}\|\theta\|_{V_{2}}^{2} d s \leq 2 e^{-2 c t}\left\|\theta_{0}\right\|_{2}^{2}+\frac{3}{c^{2}}\|Q\|_{2}^{2}
\end{gathered}
$$

and

$$
\frac{d\|u\|_{2}^{2}}{d t}+\frac{1}{2}\|u\|_{V_{1}}^{2}+\left(\frac{\lambda}{2}-c \sum_{k=1}^{m}\left|\varsigma_{k}(t)\right|^{2}\right)\|u\|_{2}^{2} \leq c A
$$


with

$$
A=\|\nabla \varsigma\|_{3}^{2}\|\varsigma\|_{2}^{2}+\|\theta\|_{2}^{2}+\|\varsigma\|_{2}^{2}+\left\|A_{1} \varsigma\right\|_{2}^{2},
$$

by Gronwall's lemma for (4.23),

$$
\begin{aligned}
\|u(t)\|_{2}^{2} \leq\|u(0)\|_{2}^{2} \exp \left(-\int_{0}^{t}\left(\frac{\lambda}{2}-c \sum_{k=1}^{m}\left|\varsigma_{k}(\sigma)\right|^{2}\right) d \sigma\right) \\
\quad+\exp \left(-\int_{0}^{t}\left(\frac{\lambda}{2}-c \sum_{k=1}^{m}\left|\varsigma_{k}(\tau)\right|^{2}\right) d \tau\right) \int_{0}^{t} A(\sigma) d \sigma .
\end{aligned}
$$

Since the process $\varsigma_{k}, k=1, \ldots, m$ is stationary and ergodic, thus we know form the ergodic theorem that

$$
\frac{1}{t} \int_{0}^{t} \sum_{j=1}^{m}\left|\varsigma_{j}(\sigma)\right|^{2} d \sigma \rightarrow E\left(\sum_{j=1}^{m}\left|\varsigma_{j}(0)\right|^{2}\right), \quad t \rightarrow \infty,
$$

thus there exists $t_{1}(\omega)$ such that any $t>t_{1}(\omega)$,

$$
\begin{aligned}
\frac{1}{t} \int_{0}^{t} \sum_{j=1}^{m}\left|\varsigma_{j}(\sigma)\right|^{2} d \sigma & \leq 2 E\left(\sum_{j=1}^{m}\left|\varsigma_{j}(0)\right|^{2}\right) \\
& \leq \frac{m}{\delta} .
\end{aligned}
$$

Takeing $\delta$ large enough so that

$$
c \int_{0}^{t} \sum_{j=1}^{m}\left|\varsigma_{j}(\sigma)\right|^{2} d \sigma \leq \frac{\lambda}{4} t
$$

As in $[\mathbf{1 1}], A(t)$ grow at most polynomially, thus we deduce

$$
\begin{aligned}
& \lim _{t \rightarrow \infty} \exp \left(-\int_{0}^{t}\left(\frac{\lambda}{2}-c \sum_{k=1}^{m}\left|\varsigma_{k}(\tau)\right|^{2}\right) d \tau\right) \int_{0}^{t} A(\sigma) d \sigma \\
& \leq \lim _{t \rightarrow \infty} \exp \left(-\frac{\lambda}{4} t\right) \int_{0}^{t} A(\sigma) d \sigma=0
\end{aligned}
$$

thus $\exists t_{2}(w)$, for $t>t_{2}(w)$, we have

$$
\exp \left(-\int_{0}^{t}\left(\frac{\lambda}{2}-c \sum_{k=1}^{m}\left|\varsigma_{k}(\tau)\right|^{2}\right) d \tau\right) \int_{0}^{t} A(\sigma) d \sigma \leq 1
$$

Taking $t_{0}(w)=\max \left(t_{1}, t_{2}\right)$, for $t>t_{0}(w)$ and $u_{0} \in \mathcal{A}(w)$, we have

$$
\|u(t)\|_{2}^{2} \leq\|u(0)\|_{V_{1}}^{2}+1 \leq R_{0}(w)+1
$$

Applying again Gronwall's lemma for (4.23) with $0 \leq t \leq t_{0}$ and noticing (4.26), we get, for $\forall t>0$

$$
\|u(t)\|_{2}^{2} \leq C_{0}(w) .
$$

Moreover, we integrate (4.23) on $[t, t+1]$ and applying (4.5),

$$
\begin{aligned}
\int_{t}^{t+1}\|u(s)\|_{V_{1}}^{2} d s & \leq 2\|u(t)\|_{2}^{2}+C \int_{t}^{t+1} \sum_{j=1}^{m}\left|\varsigma_{j}(\sigma)\right|^{2}\|u(\sigma)\|_{2}^{2} d \sigma+C \int_{t}^{t+1} A(s) d s \\
& \leq C_{1}\left(R_{0}(w), M, Q\right) .
\end{aligned}
$$


Then we will use the uniform Gronwall lemma to obtain the time-uniform estimates for $\|\widetilde{u}\|_{6}^{6}$, in $[\mathbf{1 1}]$, we have got

$$
\frac{d}{d t}\|\widetilde{u}\|_{6}^{2} \leq H(t)\|\widetilde{u}\|_{6}^{2}+c\|\theta\|_{6}^{2}
$$

with

$$
H(t)=c\left(1+\|u\|_{2}^{2}\|u\|_{V_{1}}^{2}+\|\varsigma\|_{2}^{2}\|\varsigma\|_{V_{1}}^{2}+\|u\|_{V_{1}}^{2}\right) .
$$

Since (4.27), (4.28), we get

$$
\begin{aligned}
& \int_{t}^{t+1}\|\widetilde{u}\|_{6}^{2} d s \leq \int_{t}^{t+1}\|u\|_{V_{1}}^{2} d s \leq C_{2}(w), \\
& \int_{t}^{t+1} H(s) d s \leq C_{3}\left(R_{0}(w), M, Q\right), \\
& \int_{t}^{t+1}\|\theta\|_{6}^{2} \leq \int_{t}^{t+1}\|\theta\|_{V_{2}}^{2} \leq C_{4}\left(R_{0}(w), Q\right) .
\end{aligned}
$$

By uniform Gronwall lemma we obtain $\|\widetilde{u}\|_{6}^{2} \leq C_{5}(w)$.

Then we estimate steps one steps as in [11] and by the uniform gronwall lemma, we are not difficulty to obtain the time-uniform estimates for $\|u\|_{V_{1}}^{2},\|\theta\|_{V_{2}}^{2} \leq C_{6}(w)$ and $\int_{0}^{t}\left|\Delta_{3} v(\tau)\right|_{L^{2}}^{2}+\left|\Delta_{3} \theta(\tau)\right|_{L^{2}}^{2} d \tau \leq C_{7}(w) t$, where $C_{6}, C_{7}$ are random and finite $\mathbb{P}$-a.s..

Recalling the notations for $\Gamma_{d}(t, \cdot)$, and (4.19), (4.20), we get,

$$
\begin{aligned}
\Gamma_{d}(t, w) & \leq \exp \left(-d^{\frac{4}{3}}|\Omega|^{\frac{2}{3}} \frac{1}{2 \kappa_{2}} t+d\left(|\Omega|^{\frac{1}{3}}+1\right) t+C_{6}+C_{7} t\right) \\
& \leq \exp \left(\left(-C_{8}(w) d^{\frac{4}{3}}+C_{9}(w)\right) t+C_{10}(w)\right) .
\end{aligned}
$$

Let $d$ sufficiently large, we have $\left\|\Gamma_{d}(t, w)\right\|_{L^{\infty}(\Omega)}<1$ for $\mathbb{P}$-a.s..

\subsection{Finite Dimension of the Attractor.}

THEOREM 4.4. The Hausdorff dimension of the global attractor for the SPEs is finite.

Proof. From (4.31) we get existence of some $d$ such that

$$
\left\|\Gamma_{d}(t, \cdot)\right\|_{L^{\infty}}<1 .
$$

This is the first basic estimate we need to apply Theorem 2.2 .

From the proof of (4.31), we get for the particular case $d=1$,

$$
\left\|U_{1}\right\|_{V} \leq\left\|\xi_{1}\right\|_{V} e^{C_{9} t+C_{10}} .
$$

Thus also the assumption $\gamma_{1}(t) \in L^{\infty}(\Omega, \mathbb{P})$ is fulfilled. Finally, we have to prove that $g_{\delta}(t)$ convergence to 0 in $L^{\infty}(\Omega, \mathbb{P})$ for each $t>0$.

Let $x \in V=V_{1} \times V_{2}$ and $x_{0} \in \mathcal{A}(w)$ be given, and put $\varphi_{1}(t)=\left(v_{1}, \theta_{1}\right)=$ $S(t, w) x, \varphi_{2}(t)=\left(v_{2}, \theta_{2}\right)=S(t, w) x_{0}, \varphi_{2}(t)=\left(v_{2}, \theta_{2}\right)=S(t, w) x_{0}, \varphi_{3}(t)=$ 
$\left(\varrho_{1}, \psi_{1}\right)=D_{x_{0}} S(t, w)\left(x-x_{0}\right)$, and $\eta(t)=\left(\varrho_{2}, \psi_{2}\right)=\varphi_{1}(t)-\varphi_{2}(t)-\varphi_{3}(t)$, then

$$
\begin{aligned}
& \frac{\partial\left(v_{1}-v_{2}\right)}{\partial t}+L_{1}\left(v_{1}-v_{2}\right)+\mathcal{B}_{1}\left(v_{1}, v_{1}-v_{2}\right)+\mathcal{B}_{1}\left(v_{1}-v_{2}, v_{2}\right) \\
& \quad-\int_{-h}^{z} \nabla\left(\theta_{1}-\theta_{2}\right) d \xi+f\left(v_{1}-v_{2}\right)^{\perp}=0, \\
& \frac{\partial\left(\theta_{1}-\theta_{2}\right)}{\partial t}+L_{2}\left(\theta_{1}-\theta_{2}\right)+\mathcal{B}_{2}\left(v_{1}, \theta_{1}-\theta_{2}\right)+\mathcal{B}_{2}\left(v_{1}-v_{2}, \theta_{2}\right)=0,
\end{aligned}
$$

and

$$
\begin{aligned}
& \frac{\partial \varrho_{1}}{\partial t}+L_{1} \varrho_{1}+\mathcal{B}_{1}\left(\varrho_{1}, v_{2}\right)+\mathcal{B}_{1}\left(v_{2}, \varrho_{1}\right)-\int_{-h}^{z} \nabla \psi_{1} d \xi+f \varrho_{1}^{\perp}=0 \\
& \frac{\partial \psi_{1}}{\partial t}+L_{2} \psi_{1}+\mathcal{B}_{2}\left(v_{2}, \psi_{1}\right)+\mathcal{B}_{2}\left(\varrho_{1}, \theta_{2}\right)=0
\end{aligned}
$$

Hence

$$
\begin{aligned}
& \frac{\partial \varrho_{2}}{\partial t}+L_{1} \varrho_{2}+\mathcal{B}_{1}\left(\varrho_{2}, v_{2}\right)+\mathcal{B}_{1}\left(v_{2}, \varrho_{2}\right) \\
& \quad+\mathcal{B}_{1}\left(v_{1}-v_{2}, v_{1}-v_{2}\right)-\int_{-h}^{z} \nabla \psi_{2}+f \varrho_{2}=0 \\
& \frac{\partial \psi_{2}}{\partial t}+L_{2} \psi_{2}+\mathcal{B}_{2}\left(\varrho_{2}, \theta_{2}\right)+\mathcal{B}_{2}\left(v_{2}, \psi_{2}\right)+\mathcal{B}_{2}\left(v_{1}-v_{2}, \theta_{1}-\theta_{2}\right)=0
\end{aligned}
$$

where

$$
\begin{aligned}
& \mathcal{B}_{1}(u, v):=(u \cdot \nabla) v-\int_{-h}^{z} \nabla \cdot u(x, y, \xi, t) d \xi \frac{\partial v}{\partial z}, \\
& B_{2}(u, \theta):=u \cdot \nabla \theta-\int_{-h}^{z} \nabla \cdot u(x, y, \xi, t) d \xi \frac{\partial \theta}{\partial z},
\end{aligned}
$$

and

$$
\begin{aligned}
\left\langle\mathcal{B}_{1}(u, v), w\right\rangle & =\mathfrak{b}_{1}(u, v, w), \\
\left\langle\mathcal{B}_{2}(u, \theta), \eta\right\rangle & =\mathfrak{b}_{2}(u, \theta, \eta) .
\end{aligned}
$$

Therefore

$$
\begin{aligned}
& \frac{1}{2} \frac{\partial\left\|\varrho_{2}\right\|_{V_{1}}^{2}}{\partial t}+\left\|L_{1} \varrho_{2}\right\|_{2}^{2}+\mathfrak{b}_{1}\left(\varrho_{2}, v_{2}, \Delta_{3} \varrho_{2}\right)+\mathfrak{b}_{1}\left(v_{2}, \varrho_{2}, \Delta_{3} \varrho_{2}\right) \\
& \quad+\mathfrak{b}_{1}\left(v_{1}-v_{2}, v_{1}-v_{2}, \Delta_{3} \varrho_{2}\right)+\int_{\Omega}\left(\int_{-h}^{z} \nabla \psi_{2} d \xi\right) \Delta_{3} \varrho_{2} d \Omega=0, \\
& \frac{1}{2} \frac{\partial\left\|\psi_{2}\right\|_{V_{2}}^{2}}{\partial t}+\left\|L_{2} \psi_{2}\right\|_{2}^{2}+\mathfrak{b}_{2}\left(\varrho_{2}, \theta_{2}, \Delta_{3} \psi_{2}\right)+\mathfrak{b}_{2}\left(v_{2}, \psi_{2}, \Delta_{3} \psi_{2}\right) \\
& \quad+\mathbb{b}_{2}\left(v_{1}-v_{2}, \theta_{1}-\theta_{2}, \Delta_{3} \psi_{2}\right)=0,
\end{aligned}
$$

by the Hölder, Sobolev inequality and Lemma 4.2 in [14], we get

$$
\begin{aligned}
& \frac{\partial\left(\left\|\varrho_{2}\right\|_{V_{1}}^{2}+\left\|\psi_{2}\right\|_{V_{2}}^{2}\right)}{\partial t}+\frac{1}{2}\left(\left\|\Delta_{3} \varrho_{2}\right\|_{2}^{2}+\left\|\psi_{2}\right\|_{2}^{2}\right) \\
& \quad \leq C\left(1+\left\|v_{2}\right\|_{V_{1}}^{4}+\left\|v_{2}\right\|_{V_{1}}^{2}\left\|\Delta_{3} v_{2}\right\|_{2}^{2}+\left\|\theta_{2}\right\|_{V_{2}}^{2}\left\|\Delta_{3} \theta_{2}\right\|_{2}^{2}\right)\left(\left\|\varrho_{2}\right\|_{V_{1}}^{2}+\left\|\psi_{2}\right\|_{V_{2}}^{2}\right) \\
& \quad+C\left(\left\|v_{1}-v_{2}\right\|_{V_{1}}^{3}\left\|\Delta_{3}\left(v_{1}-v_{2}\right)\right\|_{2}+\left\|v_{1}-v_{2}\right\|_{V_{1}}^{2}\left\|\theta_{1}-\theta_{2}\right\|_{V_{2}}\left\|\Delta_{3}\left(\theta_{1}-\theta_{2}\right)\right\|_{2}\right) .
\end{aligned}
$$


By the Gronwall lemma and (4.20),

$$
\begin{aligned}
\|\eta(t)\|_{V}^{2} & \leq C_{11}(t, w) \int_{0}^{t}\left(\left\|v_{1}-v_{2}\right\|_{V_{1}}^{3}\left\|\Delta_{3}\left(v_{1}-v_{2}\right)\right\|_{2}\right. \\
& \left.+\left\|v_{1}-v_{2}\right\|_{V_{1}}^{2}\left\|\theta_{1}-\theta_{2}\right\|_{V_{2}}\left\|\Delta_{3}\left(\theta_{1}-\theta_{2}\right)\right\|_{2}\right) d s .
\end{aligned}
$$

As we obtain (4.40), concerning $\varphi_{1}-\varphi_{2}$, denoting

$g(t)=1+\left\|v_{1}\right\|_{V_{1}}^{2}\left\|\Delta_{3} v_{1}\right\|_{V_{1}}^{2}+\left\|v_{2}\right\|_{V_{1}}^{2}\left\|\Delta_{3} v_{2}\right\|_{V_{1}}^{2}+\left\|\theta_{1}\right\|_{V_{2}}^{2}\left\|\Delta_{3} \theta_{1}\right\|_{V_{2}}^{2}+\left\|\theta_{2}\right\|_{V_{2}}^{2}\left\|\Delta_{3} \theta_{2}\right\|_{V_{2}}^{2}$, we finally obtain,

$$
\begin{aligned}
\left\|\varphi_{1}(t)-\varphi_{2}(t)\right\|_{V}^{2} \leq & \exp \left[C \int _ { 0 } ^ { t } \left(1+\left\|v_{1}\right\|_{V_{1}}^{2}\left\|\Delta_{3} v_{1}\right\|_{V_{1}}^{2}+\left\|v_{2}\right\|_{V_{1}}^{2}\left\|\Delta_{3} v_{2}\right\|_{V_{1}}^{2}\right.\right. \\
& \left.\left.+\left\|\theta_{1}\right\|_{V_{2}}^{2}\left\|\Delta_{3} \theta_{1}\right\|_{V_{2}}^{2}+\left\|\theta_{2}\right\|_{V_{2}}^{2}\left\|\Delta_{3} \theta_{2}\right\|_{V_{2}}^{2}\right) d s\right]\left\|x-x_{0}\right\|_{V}^{2} \\
\leq & C_{12}(t, w)\left\|x-x_{0}\right\|_{V}^{2}
\end{aligned}
$$

Consequently, we have

$$
\begin{aligned}
\int_{0}^{t}\left\|\Delta_{3}\left(\varphi_{1}-\varphi_{2}\right)\right\|^{2} d s & \leq\left\|x-x_{0}\right\|_{V}^{2}+\int_{0}^{t} g(s)\left\|\varphi_{1}(s)-\varphi_{2}(s)\right\|_{V}^{2} d s \\
& \leq C_{13}(t, w)\left\|x-x_{0}\right\|_{V}^{2} .
\end{aligned}
$$

Inserting these estimates in (4.40), we finally obtain

$$
\|\eta(t)\|_{V}^{2} \leq C_{14}(t, w)\left\|x-x_{0}\right\|_{V}^{4}
$$

where $C_{14}(t, w)$ depending on $t$ is random and finite $\mathbb{P}$-a.s.. Recalling the definition of $g_{\delta}(t)$, we see that it converges to 0 in $L^{\infty}(\Omega, \mathbb{P})$ for each $t>0$. Thus Theorem 2.2 applies.

Acknowledgements. The authors are indebted to the referee for giving some important suggestions which improved the presentations of this paper.

\section{References}

[1] Y. Cao, E. M. Lunasin, E. S. Titi, Global well-posedness of the three dimensional viscous and inviscid simplified Bardina turbulence models, Communications in Mathematical Sciences, 4 (2006), 823-84

[2] P. Constantin, C. Foias, Navier-Stokes Equations, Chicago Lectures in Mathematics, University of Chicago Press, Chicago, IL, 1988.

[3] P. Constantin, C. Foias, R. Temam, Attractors representing turbulent flows, Mem. Amer. Math. Soc., 531985.

[4] H. Crauel, A. Debussche, F. Flandoli, Random attractors, J. Dynam. Differential Equations, 9 (1997), 307-341.

[5] H. Crauel, F. Flandoli, Attractors for random dynamical systems, Probab. Theory Related Fields, 100 (1994), 365-393.

[6] H. Crauel, F. Flandoli, Hausdorff Dimension of Invariant Sets for Random Dynamical Systems, Journal of Dynamics and Differential Equations, 10 (1998), 449-474.

[7] G. Da Prato, J. Zabczyk, Stochastic Equations in Infinite Dimensions, Cambridge University Press, 1992.

[8] A. Debussche, On the finite dimensionality of random attractors, Stochastic Anal. Appl., 15 (1997), 473-491.

[9] A. Debussche, Hausdorff dimension of a random invariant set, J. Math. Pures Appl., 77 (1998), 967-988. 
[10] F. Flandoli, J.A. Langa, Determining modes for dissipative random dynamical systems, Stoch. and Stoch. Rep., 66 (1998), 1-25.

[11] H. Gao and C. Sun, Random Attractor for the $3 D$ viscous stochastic primitive equations with additive noise, Stochastics and Dynamics, 9 (2009), 293-313.

[12] H. Gao, C. Sun, Random Dynamics of the 3D Stochastic Navier-Stokes-Voight Equations, submitted.

[13] B. Guo, D. Huang, 3D Stochastic Primitive Equations of the Large-Scale Ocean: GlobalWellPosedness and Attractors, Commun. Math. Phys., 286 (2009), 697-723.

[14] N. Ju, The global attractor for the solutions to the $3 \mathrm{~d}$ viscous primitive equations, Discrete Contin. Dyn. Syst., 17 (2007), 159-179.

[15] V. K. Kalantarov, Attractors for some nonlinear problems of mathematical physics, Zap. Nauchn. Sem. Leningrad. Otdel. Mat. Inst. Steklov. (LOMI), 152 (1986), 50-54.

[16] V. K. Kalantarov, Global Behavior of Solutions of Nonlinear Equations of Mathematical Physics of Classical and Non-classical Type, Postdoctoral Thesis, St. Petersburg, 1988.

[17] V. K. Kalantarov, B. Levant, E. S. Titi, Gevrey regularity of the global attractor of the 3D Navier-Stokes-Voight equations, arXiv:0709.3328v1.

[18] V. K. Kalantarov, E. S. Titi, Global attractors and determining modes for the $3 D$ NavierStokes-Voight equations, arXiv:0705.3972.

[19] J. A. Langa, Finite-dimensional limiting dynamics of random dynamical systems, Dynamical Systems, 18 (2003), 57-68.

[20] A. P. Oskolkov, The uniqueness and solvability in the large of boundary value problems for the equations of motion of aqueous solutions of polymers, Zap. Naucn. Sem. Leningrad. Otdel. Mat. Inst. Steklov. (LOMI), 38 (1973), 98-136.

[21] B. L. Rozovskii, Stochastic Evolution Equations, Kluwer Academic Publishers, Boston, 1990.

[22] R. Temam, Infinite-dimensional Dynamical Systems in Mechanics and Physics, Springer, New York, 1997.

[23] E. Waymire, J. Duan (Eds.), Probability and Partial Differential Equations in Modern Applied Mathematics. Springer-Verlag, New York, 2005.

Institute of Mathematics, School of Mathematical Science, Nanjing Normal University, Nanjing 210046, China; and Department of Applied Mathematics, Nanjing Economics and Finance University, Nanjing, Jiangsu, China

Institute of Mathematics, School of Mathematical Science, Nanjing Normal University, Nanjing 210046, China; and Center of Nonlinear Science, Nanjing University, NANJing 210093, CHINA

E-mail address: gaohj@njnu.edu.cn 